\title{
A minimal cytomegalovirus intron A variant can improve transgene expression in different mammalian cell lines
}

\author{
L. S. Quilici • I. Silva-Pereira • A. C. Andrade • \\ F. C. Albuquerque $\cdot$ M. M. Brigido •
}

A. Q. Maranhão

Received: 12 June 2012/ Accepted: 21 August 2012/Published online: 7 September 2012

(C) Springer Science+Business Media B.V. 2012

\begin{abstract}
The expression enhancement by cytomegalovirus promoter and different intron A (IA) variants were evaluated in CHO-K1, HepG2, HEK-293 and COS-7 cells by assessing the levels of luciferase activity. This data along with mRNA levels measurement indicated that the construct harboring an IA variant with a 200 -nucleotide deletion $(\Delta 200)$ had the greatest impact on increasing luciferase expression among all constructs evaluated. Based on these results, we redesigned pCMV-IA variants and cloned them into plasmids expressing a humanized antibody. These plasmids were then used to transfect $\mathrm{CHO}-\mathrm{K} 1$ cells. Production of the antibody was not augmented with the $\Delta 200$ promoter variant. The 600 -nucleotide deletion $(\Delta 600)$ and whole IA promoter variants expressed
\end{abstract}

Electronic supplementary material The online version of this article (doi:10.1007/s10529-012-1043-z) contains supplementary material, which is available to authorized users.

L. S. Quilici · I. Silva-Pereira · F. C. Albuquerque M. M. Brigido · A. Q. Maranhão ( $₫)$ Instituto de Ciências Biológicas, Departamento de Biologia Celular, Laboratório de Imunologia Molecular, Universidade de Brasília, Bloco $\mathrm{K}, 2^{\circ}$ pavimento. Campus Universitário Darcy Ribeiro, Asa Norte, Brasília, DF 70910-000, Brazil

e-mail: andreaqm@unb.br

A. C. Andrade

Empresa Brasileira de Pesquisa Agropecuária (EMBRAPA), Centro Nacional de Pesquisa de Recursos Genéticos e Biotecnologia, Brasília, DF 70770-900, Brazil similar levels of the recombinant protein. These data indicate that the IA-based enhanced expression of transgenes depends on a small region within the intron.

Keywords CMV promoter - Gene expression · Intron A $\cdot$ Recombinant antibody

\section{Introduction}

The efficient production of heterologous proteins in animal cells depends on many factors involved in mRNA transcription and protein translation including transcription initiation, mRNA processing, nuclear translocation of mRNA, ribosome recognition and initiation of protein translation. The immediate early promoter from cytomegalovirus (pCMV) is the most popular promoter for recombinant protein expression due to its high efficiency in a broad range of cell types (Xu et al. 2001). This promoter is involved in the expression of genes in the initial stages of virus infection, thus the promoter is less dependent on tissue-specific factors. Its potency can be further enhanced by the presence of its downstream intron A (IA) (Campos-da-Paz et al. 2008; Xia et al. 2006). Intronic sequences may function in several ways: by enhancing RNA polymerase II processivity (Kwek et al. 2002), by promoting the interaction between splicing proteins and certain transcription factors and/ or proteins from RNA metabolism complexes, and by connecting and facilitating multiple types of RNA 
processing mechanisms (Le Hir et al. 2003). Introns may also contribute to translation, as splicing efficiency is inversely proportional to nonsense-mediated decay (Gudikote et al. 2005).

The wild-type IA from cytomegalovirus has been used in expression plasmid vectors for more than a decade (Mariati Ho et al. 2010; Chapman et al. 1991). However, its structure and the regulatory regions that underlie its function have yet to be mapped. With the goals of identifying a minimal enhancer element and improving recombinant antibody (ab) expression levels in mammalian cells, we constructed two truncated versions of IA and tested their efficiency by measuring the mRNA and protein levels of the firefly luciferase reporter gene. We have also tested the ability of these constructs to produce a humanized $\mathrm{ab}$ fragment (Andrade et al. 2000).

\section{Materials and methods}

\section{Plasmid constructs}

Luciferase reporter expression vectors were generated from the fluc reporter gene pGL4.14 plasmid (Promega, Madison, WI, USA). pCMV and IA were isolated from the pCMVLacI vector (Stratagene, West Cedar Creek, TX, USA) and cloned between the BglII and HindIII sites of pGL4.14. This vector was named pGLCMVIA. To produce deletion in IA, the pGLCMVIA plasmid was digested with HindIII and $X \mathrm{cmI}$ $(\Delta 600)$, HindIII and BspEI $(\Delta 400)$ and $X c m I$ and $B s p \mathrm{EI}(\Delta 200)$. The three plasmids were re-ligated after Klenow treatment.

A synthetic version of the CMV IA promoter region was designed to remove undesirable restriction sites and to ensure that the splicing acceptor site was preserved. All plasmids harboring this novel synthetic pCMV were named pCO. The synthetic promoters were used to direct the expression of a recombinant anti-CD3 ab fragment. For the ab construct, the full IA/promoter was cloned into an ab expression vector (pMIRES) (Silva et al. 2009) using the XmaI and SbfI restriction sites (pCOAb). From this vector, the truncated IA variants were obtained using either a single $B s p$ EI digestion $(\Delta 200)$ or a $B s p E I / N r u I$ doubledigestion $(\Delta 600)$ followed by Klenow treatment. These plasmids were named pCOAb $\Delta 200$ and pCOAb $\Delta 600$, respectively. The transgene coded by
pCO plasmids is an ab $\mathrm{FvFc}$ fragment (Andrade et al. 2000). This fragment is a scFv (VH-linker peptide-VL) connected to a human IgG1 Fc ( $\mathrm{CH} 2 \mathrm{CH} 3$ domains).

Cell culture and transfection

CHO-K1 (ATCC CCL-61), HepG2 (ATCC HB-8065), HEK-293 (ATCC CRL-1593) and COS-7 (ATCC CRL-1651) cells were grown at $37{ }^{\circ} \mathrm{C}$ in $5 \% \mathrm{CO}_{2}$ and $70 \%$ humidity, in appropriate culture medium. Transfections were performed with lipofectamine 2000 (Invitrogen) according to the manufacturer's instructions. The control plasmid pGL4.73, containing Renilla luciferase (rluc), was co-transfected with the test plasmids at a ratio of 1:10. For all constructs, the transfection assays were performed in triplicate for three independent experiments.

For ab plasmid transfection, $6 \times 10^{5}$ cells were seeded on a 6-well plate and transfected using a mixture of $4 \mu \mathrm{g}$ DNA and $10 \mu \mathrm{l}$ lipofectamine. For transient transfection, the supernatants were collected $48 \mathrm{~h}$ later.

\section{Luciferase activity assays}

Measurements of Fluc and Rluc activities were performed using the dual-luciferase Reporter Assay System Kit (Promega, Madison, WI, USA) according to the manufacturer's instructions. All assays were carried out using a TD 20/20 luminometer (Turner Designs). Data points measured the ratio of Fluc to Rluc activity. We performed ANOVA followed by a Tukey post hoc test to evaluate differences among the groups. All statistical analyses were performed with MS-Excel and MINITAB release 14.20 (Minitab Inc).

Total RNA extraction and cDNA synthesis

After 24-48 h transfection, total RNA was extracted from the CHO-K1 cells cultured in 6-well plates using Trizol. The RNA was treated with RNAse-free DNAse I (Promega) for $45 \mathrm{~min}$ at $37^{\circ} \mathrm{C}$ and then precipitated with ethanol. The samples were quantified with a Gene Quant spectrophotometer and analyzed on an RNAsefree $1 \%$ agarose gel. Subsequently, cDNA synthesis was performed using $2 \mu \mathrm{g}$ treated RNA, Superscript III reverse transcriptase (Invitrogen) and oligo-dT as a primer according to the manufacturer's instructions. For qPCR experiments (see following "qPCR"), $2 \mu \mathrm{l}$ diluted cDNA (1:10) was used. As a control, we 
performed the reaction without reverse transcriptase. A DNA contamination control was also performed by carrying out a reaction without reverse transcriptase.

\section{qPCR}

Quantitative PCR (qPCR) assays were performed using a 7500 Fast Real-Time PCR System (Applied Biosystems) to measure $f l u c$, rluc and ab transgene mRNA levels. The results were analyzed using 7500 Fast System SDS Software. The cycling parameters were as follows: stage $1,50{ }^{\circ} \mathrm{C}$ for $2 \mathrm{~min}$ and $95^{\circ} \mathrm{C}$ for $5 \mathrm{~min}$; and stage $2,95{ }^{\circ} \mathrm{C}$ for $3 \mathrm{~s}$ and $60{ }^{\circ} \mathrm{C}$ for $30 \mathrm{~s}$. The second stage was repeated 40 times. The primer sequences used for the fluc, rluc, hamster GAPDH, and heavy (VH) and light (VL) anti-CD3 ab chains and the unprocessed CMV exon 1 genes are listed in Supplementary Table 1. The qPCR reactions were performed using the Platinum SYBR Green qPCR SuperMix-UDG Kit (Invitrogen) in $10 \mu$ l. The primers were used at $2 \mathrm{mM}$. The $2^{\Delta \Delta \mathrm{Ct}}$ method was used for calculating mRNA transcript levels, as previously described (Bubner et al. 2004).

\section{ELISA}

Antibody production was measured in culture supernatants using a sandwich-ELISA assay, as described in Silva et al. (2009). Briefly, microtiter plates were coated with goat anti-human IgG H + L (Thermo Scientific-Pierce) diluted 1:1,000 in PBS. After three wash steps with PBS, plates were blocked with $5 \%$ nonfat milk diluted in PBST $(0.05 \%$ Tween 20). Next, the supernatants from the transient transfections were added in 1:1, 1:3 and 1:9 proportions. After three wash steps, a goat anti-human Fc ab conjugated to alkaline phosphatase (Sigma) $(1: 5,000$ dilution) was added. Finally, $p$-nitro-phenylphosphate at $2 \mathrm{mg} / \mathrm{ml}$ was used as a chromogenic substrate. The absorbance was measured at $405 \mathrm{~nm}$ using a microplate reader. The protein concentration was estimated based on the human IgG standard curve.

\section{Results}

IA and variants increased luciferase activity

The novel luciferase reporter plasmid constructs harboring truncated versions of CMV IA are given in Fig. 1. These vectors were transfected into four mammalian cell lines: CHO-K1, COS-7, HepG2 and HEK-293. These cell lines represent the major cell lineages used for biotechnological purposes and were used to evaluate heterologous protein production from the newly developed constructs. Following transfection, luciferase activity was measured. The Fluc activity was normalized to the Rluc activity to control for transfection efficiency. The relative luciferase activity for each cell line is shown in Fig. 2 (panels a-d). Among the cell lines, HEK-293 exhibited the highest luciferase activity, at least 2,500\% greater activity than the other cell lines (Fig. 2, panel d). Analysis of the luciferase expression levels indicated that the IA deletions had similar effects on all of the cell lines, although the maximum luciferase activities differed among cell lines. The pGLCMV $\Delta 200$ construct produced the highest luciferase activity (up to $200 \%$ greater than the IA construct) in all of the cell lines (Fig. 2). In contrast, the $\Delta 600$ construct had a variable effect: it increased the luciferase activity 100-150\% in CHO-K1 and HepG2 cells compared to the wild-type IA (Fig. 2, panels a, b); it produced no effect in HEK-293 cells (Fig. 2, panel d); and it slightly reduced luciferase activity in COS-7 cells (Fig. 2, panel c) relative to the wild-type IA The pGLCMV $\Delta 400$ construct exhibited the lowest luciferase activity levels for all the cell lines tested. Statistically significant data are indicated in the Fig. 2 panels.

IA variants increased luciferase mRNA levels

To investigate the correlation between luciferase activity and luciferase mRNA levels, we performed qPCR with cDNAs prepared from CHO-K1 cells transfected with pGLCMVIA, pGLCMVI $\Delta 200$ and pGLCMVI $\Delta 600$ constructs Considering that the $\Delta 400$ variant produced low levels of luciferase activity, this construct was not examined further. We also focused our experiments on $\mathrm{CHO}$ cells due to their widespread industrial use. The qPCR results are shown in Fig. 3, indicating that intact IA, along with the $\Delta 200-\Delta 600$ variants, enhanced the accumulation of luciferase mRNA in the first $24 \mathrm{~h}$ after transfection. By comparing the graphs depicted in Figs. 2a and 3, it is clear that enhanced protein activity correlates well with mRNA levels for the $\Delta 200$ construct. The $\Delta 600$ construct, however, produced comparable mRNA 


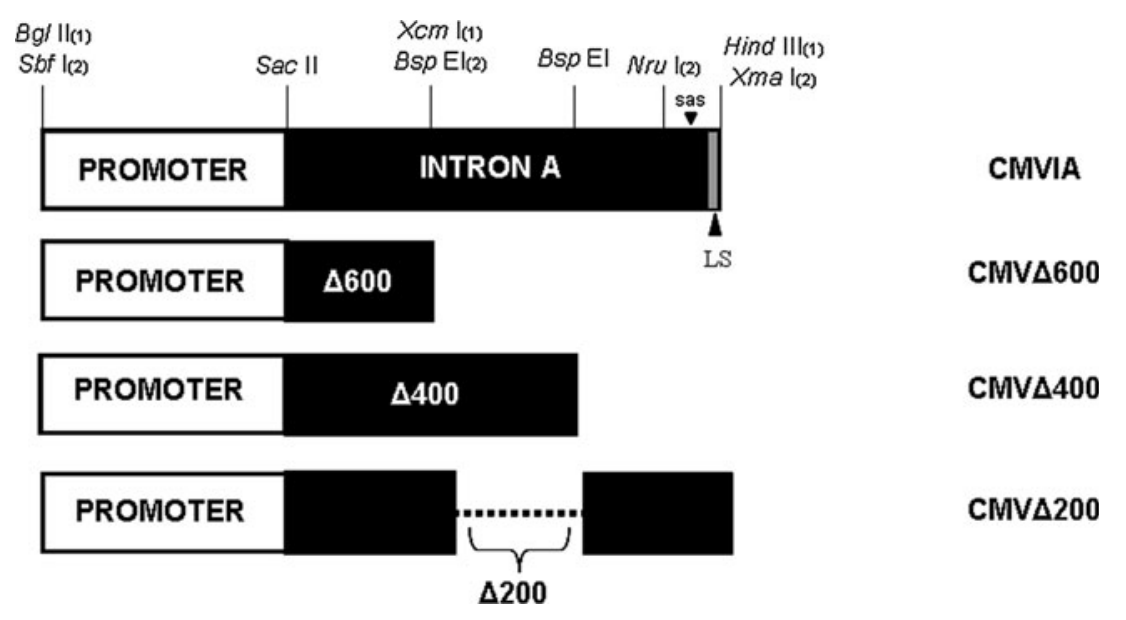

Fig. 1 Schematic representation of the original and synthetic IA constructs For constructs using the pGL4.14 vector (Promega), the promoter/IA element was obtained from the pCMVLacI vector (Stratagene) by BglII and HindIII cleavage. The deleted versions of IA were obtained by elimination of the region between $X c m$ I and HindIII, BspEI and HindIII, or XcmI and $B s p$ EI sites for IA $\Delta 600, \Delta 400$, or $\Delta 200$, respectively. The synthetic IA element was cloned between the $S b f \mathrm{I}$ and $X m a \mathrm{I}$ sites in the pMIRES vector (Silva et al. 2009). The $\Delta 600$ variant

levels to $\Delta 200$, but had lower luciferase activity after $24 \mathrm{~h}$.

The level of luciferase mRNA decreased $48 \mathrm{~h}$ after transfection (black bars in Fig. 3). This effect was observed for all constructs, but was particularly drastic for the $\Delta 600$ variant. For every 7 transcripts present at $24 \mathrm{~h}$, only one was present after $48 \mathrm{~h}$. The ratios for IA and $\Delta 200$ were $2.5: 1$ and 2:1, respectively.

Developing synthetic IA and variants that preserve acceptor splice site

Because the luciferase mRNA expression levels dropped sharply after $48 \mathrm{~h}$ for the $\Delta 600$ variant, we hypothesized that the removal of a splice acceptor site in this variant resulted in a loss of stability and consequently, lower expression efficiency (Fig. 3). To test this, we chemically synthesized the PCMV/IA construct to eliminate unnecessary restriction sites and to ensure the presence of the desired splice acceptor site in all IA variants. The synthetic IA promoters were tested for luciferase expression and exhibited mRNA levels comparable to the original IA variants, although a difference was observed in terms of luciferase activity. We observed a decrease in enzyme activity for $\Delta 200$ and an increase in activity of synthetic IA was obtained using a single $B s p \mathrm{E}$ I digestion. For the $\Delta 200$ s variant, a $N r u \mathrm{I} / B s p \mathrm{E}$ I double digestion was carried out. The leader sequence (LS) is found only on synthetic constructs expressing the recombinant ab. (1) Restriction sites used for cloning the pCMVLacI promoter and IA in the pGL4.14 plasmid. (2) Restriction sites used for cloning the synthetic IA and IA variants in the pMIRES plasmid. SAS splicing acceptor site, $L S$ leader sequence

for the $\Delta 600$ variant, as compared with the original variants (Supplementary Fig. 1).

We measured the level of unspliced CMV IE mRNA in all constructs (original and synthetic). Indeed, we found that the original $\Delta 600$ construct, which had lost its splice acceptor site, had $250 \%$ more unprocessed mRNA than the other constructs that preserved this region (Fig. 4).

Effect of IA and variants on recombinant $a b$ production and mRNA levels in CHO cells

To test the ability of IA variants to enhance secretable recombinant protein production, we constructed vectors expressing a FvFc ab fragment harboring IA, $\Delta 200$ or $\Delta 600$. This recombinant ab was a single-chain variable fragment $(\mathrm{scFv})$ connected to a human $\mathrm{IgG} 1$ constant domain (Fc) (Andrade et al. 2000). The pCMVIA synthetic gene was named pCO, and the plasmids were named pCOAb, pCOAb $\Delta 200$ and pCOAb $\Delta 600$, indicating their respective deletions. Figure 5a shows the transient expression of the humanized $\mathrm{ab}$. The intact IA and the $\Delta 600$ constructs exhibited similar levels of secretable protein production, while the $\Delta 200$ variant exhibited the lowest protein production $(50 \%$ less than the full IA 
A
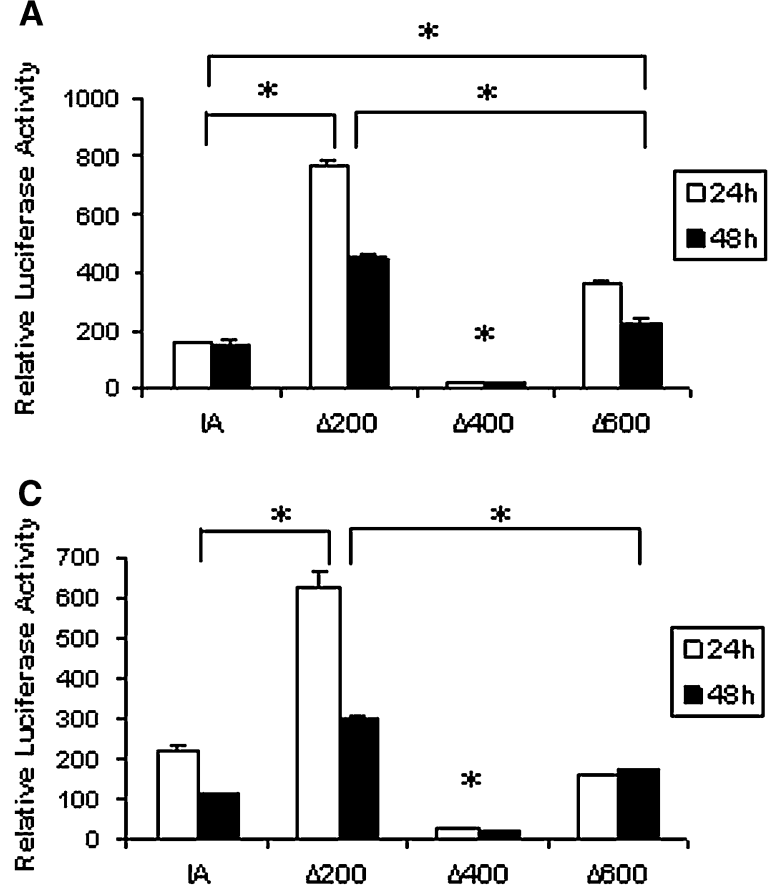

Fig. 2 Transient luciferase activity in different cell lines for the original IA constructs The relative luciferase activities (Fluc/ Rluc) of the IA constructs in CHO-K1 (panel a), HepG2 (panel b), COS-7 (panel c) and HEK-293 cells (panel d) are shown. The values, in relative luminescence units, indicate the activity for each construct relative to the activity for the intronless construct.

construct We also evaluated $\mathrm{Ab}$ production in mixed population of cells kept under genetic in pressure for 30 days. The same production pattern was observed (Supplementary Fig. 2).

The mRNA levels for the anti-CD3 ab were measured by qPCR assays (Fig. 5 b). The $\Delta 600$ variant exhibited elevated mRNA production $(\sim 300 \%$ higher than the synthetic IA construct). The $\Delta 200$ variant displayed ab mRNA production $100 \%$ greater than the intact IA construct.

\section{Discussion}

Several studies have demonstrated the importance of introns for transcription and protein expression. The IA of CMV has been widely used for biotechnological applications and is able to enhance protein production in several mammalian cell lines (Xu et al. 2001; Campos-da-Paz et al. 2008; Xia et al. 2006). The present study developed and used new CMV IA variants, which are depicted in Fig. 1.
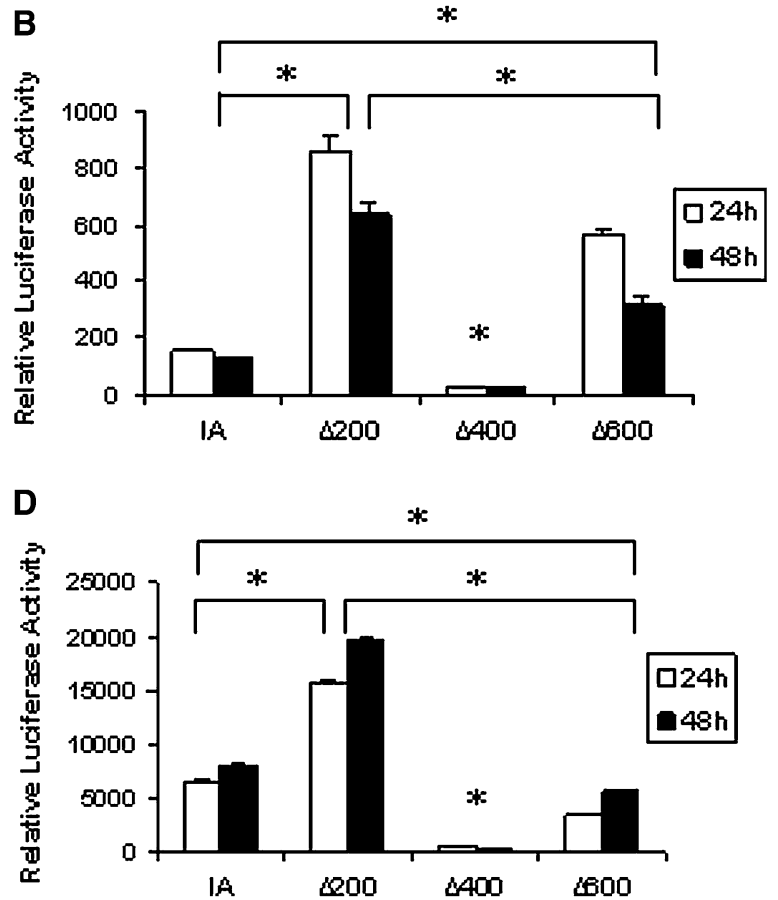

The measurements were performed $24-48 \mathrm{~h}$ after transfection. At least three independent experiments were performed in triplicate. The asterisks represent $P<0.05$, indicating a significant difference among different constructs (IA, $\Delta 200$, $\Delta 400$ and $\Delta 600)$. The $\Delta 400$ variant luciferase activity values are significantly lower considering the other constructs analyzed

The IA deletion variants along with the CMV promoter were more effective in enhancing luciferase activity than wild-type IA (Fig. 2). It is important to note the variance of luciferase activity among the cell lines tested, especially the HEK-293 cell line, which exhibited the highest luciferase activity levels. This cell line has been found to produce large amounts of recombinant protein more rapidly than $\mathrm{CHO}-\mathrm{K} 1$ cells (Thomas and Smart 2005).

Here, we demonstrate that there is a greater accumulation of the luciferase and ab mRNAs derived from the IA, $\Delta 200$ and $\Delta 600$ constructs (at $24 \mathrm{~h}$ after transfection) (Figs. 3, 5b). Furthermore, the luciferase mRNA levels correlated with the measured luciferase activity, indicating coupling of transcription and translation. Interestingly, the same correlation was not observed for ab production, as discussed below.

Our data indicate that a minimal truncated portion of IA may improve the synthesis of transgenes. Moreover, this effect may be attributed, at least in part, to an augmentation of the mRNA level (Figs. 3, 5b). In the original $\Delta 600$ variant expressing luciferase gene we 


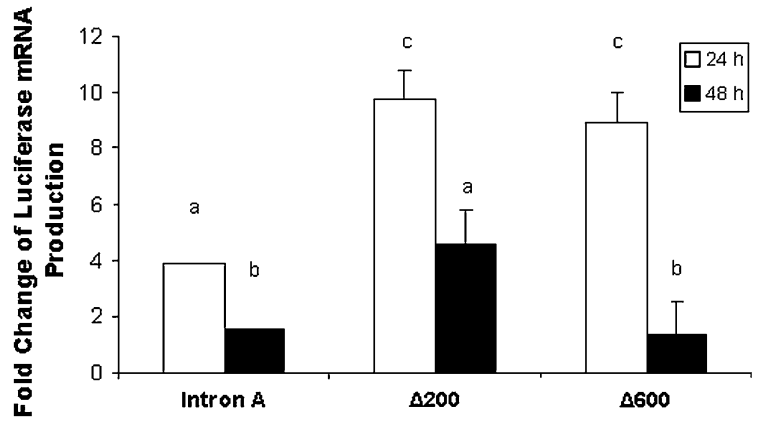

Fig. 3 Luciferase mRNA transcript levels. The mRNA levels in $\mathrm{CHO}$ cells were measured by qPCR $24-48 \mathrm{~h}$ after transfection. The increase in luciferase mRNA levels indicates the fold change relative to the intronless construct control using the $2^{\Delta \Delta \mathrm{Ct}}$ method. The letters $(a-c)$ indicate the statistical relationships among different constructs (IA, $\Delta 200$ and $\Delta 600$ ). At least three independent experiments were performed in triplicate. Different letters indicate statistically significant differences, with $P<0.05$

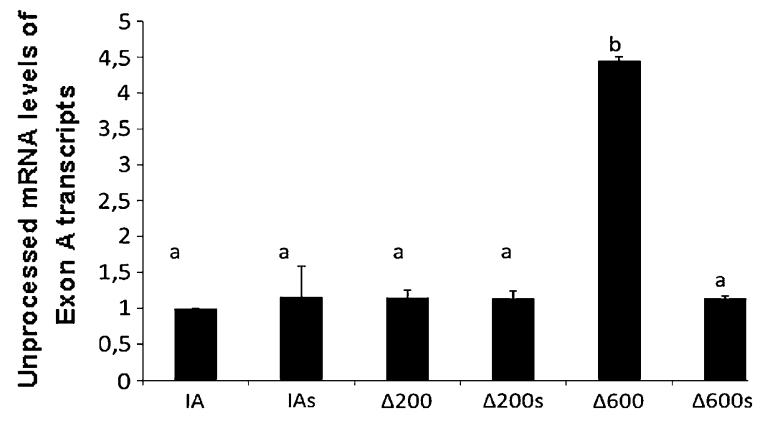

Fig. 4 CMV exon A unprocessed mRNA levels The levels of unprocessed transcripts from CMV exon A were measured in CHO-K1 cells by qPCR $24 \mathrm{~h}$ after transfection. The increase in luciferase mRNA levels indicates the fold change relative to the intronless construct control using the $2^{\Delta \Delta \mathrm{Ct}}$ method. At least three independent experiments were performed in triplicate. Different letters indicate statistically significant differences, with $P<0.05$

found a decrease in the mRNA level after $48 \mathrm{~h}$ of transfection. This effect seem to be due to the loss of the splicing acceptor site between the BspEI and HindIII sites. Although a cryptic splicing site can be used (Awasti et al. 2004), the removal of the original site may reduce the efficiency of splicing. An accumulation of unspliced mRNA may make it unstable (Jaillon et al. 2008), leading to its degradation by NMD (nonsensemediated decay) machinery (Gudikote et al. 2005). To address this possibility, we developed IA variants that maintained the splice acceptor site region. We demonstrated that there were an accumulation of
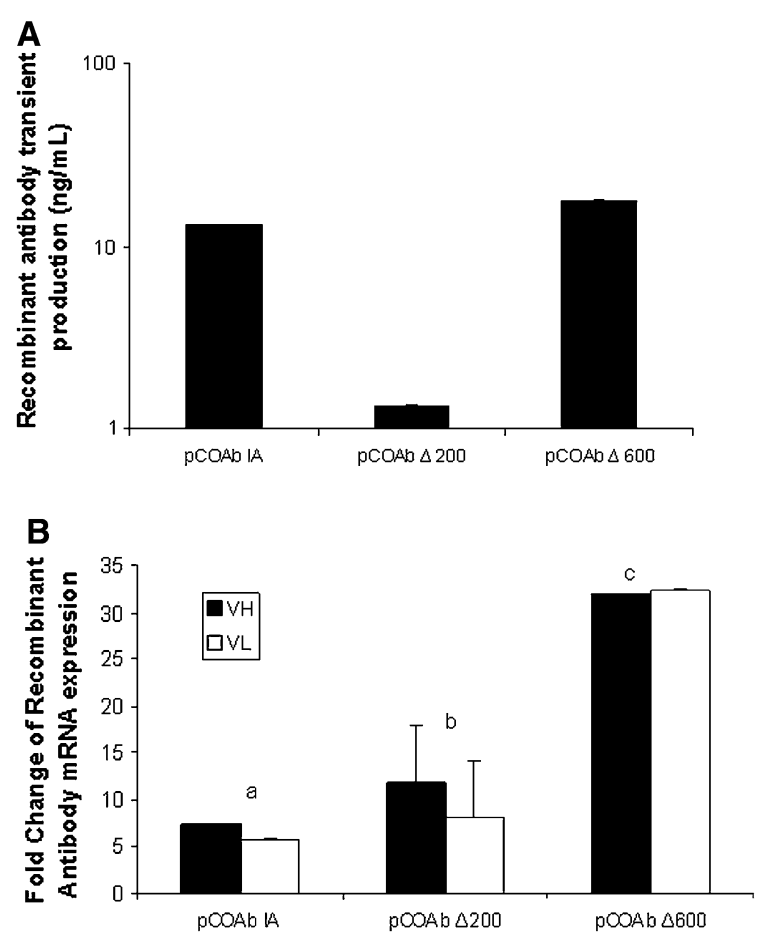

Fig. 5 Recombinant ab transient expression and mRNA production. The supernatants from transient transfections (panel a) were collected $48 \mathrm{~h}$ after transfection of $\mathrm{CHO}$ cells to calculate the amount of ab secreted. ELISA assays were performed in triplicate on two independent occasions. The ab mRNA levels in CHO cells (panel $b$ ) were measured by qPCR $24 \mathrm{~h}$ after transfection. The increase in ab mRNA indicates the fold change relative to the intronless construct control using the $2^{\Delta \Delta \mathrm{Ct}}$ method. The letters $(a-c)$ indicate the statistical relationships among different constructs. Two independent experiments were performed in triplicate. Different letters indicate statistically significant differences, with $P<0.05$

unprocessed CMV exon A mRNA on wild-type IA $\Delta 600$ variant (Fig. 4), corroborating our hypothesis.

The synthetic IA $\Delta 600$ and $\Delta 200$ deletions were further tested for the expression of the recombinant $a b$ transgene. Antibody and luciferase mRNAs levels were higher for the variants than for intact IA (Fig. 5b). No correlation was observed for Ab production, whereas protein measurements were similar between intact and $\Delta 600$ variants and smaller for the $\Delta 200$ construct (Fig. 5a). Therefore, protein expression and secretion depends on a complex set of factors and does not parallel mRNA production.

This finding is not contradictory to the generally accepted idea of coupling of transcription and translation because ab protein was not measured in the cytoplasm; rather, it was detected in cell culture 
supernatants as a mature dimeric protein. Considering that an ab is a complex molecule requiring proper assembly for secretion (Gomez et al. 2012), its production involves several components of the cell machinery. Thus, the use of a strong modified promoter may not be sufficient to achieve the expected final protein level in cell culture supernatants. In the case of ab production, transcriptional enhancement is not followed by protein production and secretion. This same effect was observed for IFN gamma and Trastuzumab heterologous expression in $\mathrm{CHO}$ cells (Mariati et al. 2010a). Thus, besides translation, ab levels are dependent on other downstream processes, including secretion, assembly and transport.

\section{Conclusion}

The CMV IA harbors regulatory elements that affect its function as an enhancer of expression, although only certain regions are necessary for the enhancement of protein production (i.e., the minimal functional intron). We showed that these effects are due to an increase in mRNA levels that can enable a more efficient translational process and that a minimal intron ( $\Delta 600$ variant) is sufficient to preserve its regulatory function. These constructs may therefore be used as valuable tools for biotechnological processes such as enhancing heterologous protein expression.

Acknowledgments We thank Izabel CristinaRodrigues Silva for assistance with the statistical analysis. This work was supported by Conselho Nacional do Desenvolvimento Científico e Tecnológico (CNPq) and Financiadora de Estudos e Projetos (FINEP).

\section{References}

Andrade EV, Albuquerque FC, Moraes LMP, Brigido MM, Santos-Silva MA (2000) Single-chain Fv with Fc fragment of the human igG1 tag: construction, Pichia pastoris expression and antigen binding characterization. J Biochem 128:891-895

Awasti S, Isler JA, Alwine JC (2004) Analysis of splice variants of the immediate-early 1 region of human cytomegalovirus. J Virol 78:8191-8200
Bubner B, Gase K, Baldwin IT (2004) Two-fold differences are the detection limit for determining transgene copy numbers in plants by real-time PCR. BMC Biotechnol 4:1-11

Campos-da-Paz M, Costa CS, Quilici LS, Simões IC, Kyaw CM, Maranhão AQ, Brigido MM (2008) Production of recombinant human Factor VIII in different cell lines and the effect of human XBP-1 co-expression. Mol Biotechnol 39:155-158

Chapman BS, Thayer RM, Vincent KA, Haigwood NL (1991) Effect of intron A from human cytomegalovirus (Towne) immediate-early gene of heterologous expression in mammalian cells. Nucleic Acids Res 19:3979-3986

Gomez N, Subramanian J, Ouyang J, Nguyen MDH, Hutchinson M, Sharma VK, Lin AA, Yuk IH (2012) Culture temperature modulates aggregation of recombinant antibody in CHO cells. Biotechnol Bioeng 109(1):125-136

Gudikote JP, Imam JS, Garcia RF, Wilkinson MF (2005) RNA splicing promotes translation and RNA surveillance. Nat Struct Mol Biol. 12:801-809

Jaillon O, Bouhouche K, Gout JF, Aury JM et al (2008) Translational control of intron splicing in eukaryotes. Nature 451:359-362

Kwek KY, Murphy S, Funger A, Thomas B, O'Gorman W, Kimura H, Proudfoot NJ, Akoulitchev A (2002) U1 snRNA associates with TFIIH and regulates transcriptional initiation. Nat Struct Mol Biol. 9:800-805

Le Hir H, Nott A, Moore MJ (2003) How introns influence and enhance eukaryotic gene expression. Trends Biochem Sci 28:215-220

Mariati Ho SCL, Yap MGS, Yang Y (2010b) Evaluating posttranscriptional regulatory elements for enhancing transient gene expression levels in CHO K1 and HEK293 cells. Protein Exp Purif. 69:9-15

Mariati Ng YK, Chao S-H, Yap MGS, Yang Y (2010a) Evaluating regulatory elements of human major immediate early gene for enhancing transgene expression levels in CHO K1 and HEK 293 cells. J Biotechnol 147:160-163

Silva HM, Vieira PMMM, Costa PLN, Pimentel BMS, Moro AM, Kalil J, Maranhão AQ, Coelho V, Brigido MM (2009) Novel humanized anti CD-3 antibodies induce a predominantly immunoregulatory profile in human peripheral blood mononuclear cells. Immunol Lett 125:129-136

Thomas P, Smart GT (2005) HEK293 cell line: a vehicle for the expression of recombinant proteins. J Pharmacol Toxicol Methods 51:187-200

Xia W, Bringmann P, McClary J, Jones PP, Manzana W et al (2006) High levels of protein expression using different mammalian CMV promoters in several cell lines. Protein Exp Purif. 45:115-124

$\mathrm{Xu}$ ZL, Mizuguchi H, Watabe AI, Uchida E, Mayumi T, Hayakawa T (2001) Optimization of transcriptional regulatory elements for constructing plasmid vectors. Gene 272:149-156 